\title{
Single-shot temperature- and pressure-sensitive paint measurements on an unsteady helicopter blade
}

\author{
Kevin J. Disotell • Di Peng • Thomas J. Juliano • \\ James W. Gregory • Jim W. Crafton • \\ Narayanan M. Komerath
}

Received: 13 June 2013/Revised: 27 December 2013/Accepted: 16 January 2014/Published online: 5 February 2014

(C) The Author(s) 2014. This article is published with open access at Springerlink.com

\begin{abstract}
Unsteady pressure-sensitive paint (PSP) measurements were acquired on an articulated model helicopter rotor of $0.26 \mathrm{~m}$ diameter in edgewise flow to simulate forward flight conditions. The rotor was operated at advance ratios (free stream velocity normalized by hover tip speed) of 0.15 and 0.30 at a cycle-averaged tip chord Reynolds number of $1.1 \times 10^{5}$, with collective and longitudinal cyclic pitch inputs of $10^{\circ}$ and $2.5^{\circ}$, respectively. A single-shot data acquisition technique allowed a camera to record the paint luminescence after a single pulse of high-energy laser excitation, yielding sufficient signal-tonoise ratio to avoid image averaging. Platinum tetra(pentafluorophenyl) porphyrin (PtTFPP) in a porous polymer/ ceramic binder served as the PSP. To address errors caused by image blurring and temperature sensitivity, a previously reported motion deblurring algorithm was implemented and the temperature correction was made using temperature-sensitive paint measurements on a second rotor blade. Instantaneous, unsteady surface pressure maps at a rotation rate of $82 \mathrm{~Hz}$ captured different aerodynamic responses between the two sides of the rotor disk and were compared to the nominally steady hover case. Cycle-to-cycle
\end{abstract}

K. J. Disotell · D. Peng · T. J. Juliano · J. W. Gregory ( $ه)$ Aerospace Research Center, Department of Mechanical and Aerospace Engineering, The Ohio State University, 2300 West Case Road, Columbus, $\mathrm{OH} 43235$, USA

e-mail: gregory.234@osu.edu

J. W. Crafton Innovative Scientific Solutions, Inc., 2766 Indian Ripple Road, Dayton, $\mathrm{OH} 45440$, USA

N. M. Komerath

Daniel Guggenheim School of Aerospace Engineering, Georgia Institute of Technology, 270 Ferst Drive, Atlanta, GA 30332, USA variations in tip unsteadiness on the retreating blade were also observed, causing oblique pressure features which may be linked to three-dimensional stall.

\section{Introduction}

The main rotor blades of a helicopter in forward flight encounter time-varying pressure changes imposed by the difference in relative flow velocity between the advancing and retreating sides of the disk. The advancing blade sees a higher blade-relative velocity in forward flight due to the added free stream velocity. This asymmetry produces cyclically varying air loads at the rotational frequency of the rotor. To accurately measure the unsteady air loads, a technique with high spatial and temporal resolution must be employed. Instrumenting a rotating blade with pressure transducers poses difficult and expensive issues in blade fabrication, balancing, extracting signals from the rotating frame and transmitting at a sufficiently high frequency. Pressure-sensitive paint (PSP) diagnostics have evolved to measure the pressure distribution on rotating blades with high spatial and temporal resolution.

Pressure-sensitive paint is an image-based measurement technique which operates on the photokinetic reaction between flowing air and a luminescent coating applied to the surface of a model, in which a molecular-sized array of chemical sensors responds to the local air pressure at the wall (Bell et al. 2001; Liu and Sullivan 2005). A typical PSP system is comprised of the painted test article, a light source for exciting the paint, and photodetector for recording the light emission from the paint. The binder matrix adheres the luminescent molecules to the model substrate. Developments in binder design have enabled kilohertz-level frequency response, making the paint 
suitable for unsteady pressure measurements (Gregory et al. 2008, 2014). At least two images of the painted surface are required to measure the pressure field, one acquired at a given reference condition ("wind-off") and the other acquired with the paint responding to the unknown pressure field ("wind-on"). The two images are aligned using conventional mapping transforms (Bell and McLachlan 1996). By phase locking the excitation source and photodetector to the rotational frequency of the rotor, the surface pressure map is obtained at desired azimuth positions.

Primary contributors to error in the PSP technique are model movement through a non-uniform illumination field and the undesirable temperature sensitivity of luminescence. These major sources of error especially complicate PSP measurements on a rotating blade, where blade deformation and viscous heating can be present. To minimize the error caused by blade deformation and non-uniform illumination fields, Watkins et al. (2007) and Wong et al. (2005) have found that the lifetime-based method of PSP data acquisition is superior to the intensity-based method in this regard. Lifetime-based PSP data acquisition is predicated upon the pressure sensitivity of the temporal decay of luminescence (with characteristic lifetime $\tau$ ) resulting from pulsed excitation. This approach differs from intensity-based methods where the light source is flashed for each wind-off and wind-on image (Liu and Sullivan 2005); instead, the reference information is obtained from the same wind-on condition as the pressure information. In practical implementation of the lifetime method, a two-gate CCD camera acquires separate images during the PSP's response (a single lifetime decay). To convert the light emission from the paint to pressure, a ratio of the two exposure gates is formed which self-references the single pulse of illumination to eliminate the effects of model movement and non-uniform excitation field. Gate 1 represents the time integral of the paint luminescence which covers the single pulse of excitation light (during which the paint shows very little pressure response, essentially serving as a reference), while Gate 2 captures the bulk of the pressure-sensitive luminescence decay after the excitation pulse. In this way, the ratio of intensity between Gate 2 and Gate $1, I_{2} / I_{1}$, is indicative of the pressure change relative to reference conditions. Figure 1 depicts this two-gate lifetime method; note that the lifetime $\tau$ is a function of local temperature $T$ and pressure $p$.

The exposure duration of Gate 1 may be adjusted on a double-framed camera to balance the light intensity in both gates at reference conditions, allowing maximum pressure sensitivity while minimizing the effects of imager shot noise. When Watkins et al. (2007) and Wong et al. (2005, 2010) applied the lifetime PSP method to rotorcraft testing, they observed less edge blurring and were able to capture

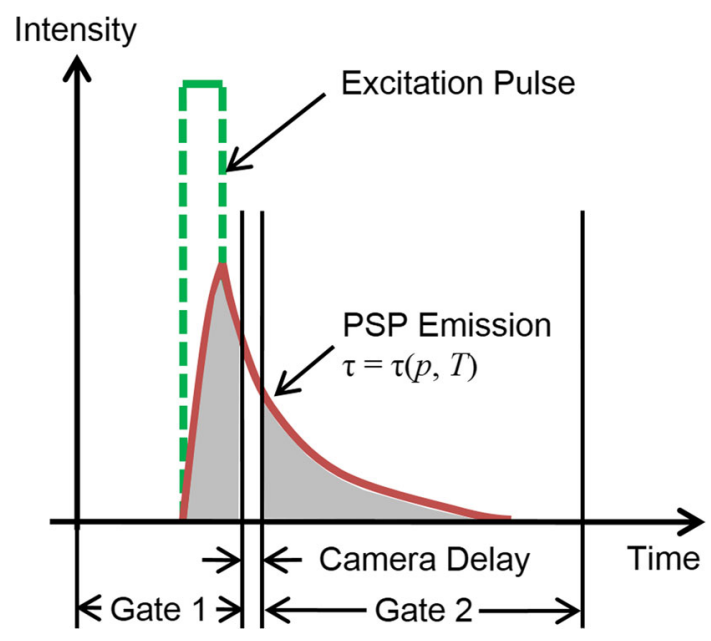

Fig. 1 Single-shot, two-gate lifetime method for PSP measurement

the qualitative trend of leading-edge suction followed by pressure recovery toward the trailing edge of the blade. However, they noted image misalignment effects caused by cycle averaging of the unsteady blade motion and blurring artifacts were not completely resolved. Sakaue et al. (2013) approached the problems of blade deformation and temperature sensitivity by employing a two-component PSP in which pressure-sensitive and pressure-insensitive luminescent molecules were mixed into a paint layer and imaged by a high-speed color camera. By ratioing the light emissions captured simultaneously from each paint component, the effects of non-uniform illumination and unsteady surface motion were cancelled. The two PSP components that are excited by the same illumination source must be carefully chosen to reduce spectral overlap of their emission wavelengths, as this reduces the pressure sensitivity of the system.

To avoid the need for phase averaging of PSP images, Kumar (2010) and Juliano et al. (2011) demonstrated that acceptable signal-to-noise ratio in a single image can be achieved with a high-energy laser providing a single shot of excitation in the lifetime method. In addition to improved signal level, the high-energy, collimated excitation enables long working distances between the illumination source and the test article. To cancel the effects of non-repeatable mode structure and speckle in the laser illumination field, a two-gate lifetime method is necessary. The utility of the single-shot technique is that it allows aperiodic, transient pressure information to be captured which would otherwise be averaged. For example, Disotell and Gregory (2011) observed superimposed wave-like structures in the acoustic field of a resonance cavity operating at high sound pressure levels using the single-shot PSP technique. This property is particularly desirable for helicopter flows, where dynamic stall and tip vortex 
behavior are routinely observed to be aperiodic phenomena (McCroskey 1981; Leishman 1998).

Recently, Wong et al. (2012) and Watkins et al. (2012) utilized the single-shot lifetime technique to acquire unsteady PSP measurements near the tip of a scale helicopter blade in simulated forward flight at an advance ratio of 0.35. Using a porous polymer/ceramic (PC) binder with platinum tetra(pentafluorophenyl) porphyrin (PtTFPP) as the luminescent molecule, the paint exhibited blurring effects near the blade edges and temperature sensitivity which could not be entirely overcome from an in situ calibration using a set of pressure transducers. The blurring problem arises from the long lifetime of the luminescent decay relative to the length of the second exposure gate, which is essentially the camera readout time of the first exposure. Blurring artifacts are most pronounced near the edges of the blade and must be corrected to uncover the critical pressure information existing in these regions, such as the signature of a leading-edge vortex. To address the issue of image blurring, Juliano et al. (2012) presented a rectilinear deconvolution filter for PSP data which was validated by experiments on a rotating disk.

In conducting a test campaign on a small-scale helicopter model following Disotell et al. (2012), the current work addresses the above error sources for PSP applied to rotating blades and demonstrates the necessary data processing steps which can be deployed in future large-scale ground testing of rotorcraft. Full-span blade pressure maps were obtained on a two-bladed, 0.256-m diameter rotor. Following a description of the experimental apparatus, details on the data reduction and correction procedures are presented, including a temperature correction method using temperature-sensitive paint (TSP) applied to a second blade. The resulting surface pressure maps are compared at two different advance ratios $(\mu=0.15$ and 0.30$)$ as well as several blade positions on the rotor disk: advancing and retreating blades with the free stream approximately aligned in the chordwise direction, and a blade with crossflow near the front of the disk. The structure of the unsteadiness is revealed by inspecting full-field maps of root-mean-square pressure fluctuations constructed from the ensemble of single-shot images. A complementary ACcoupled view of differential pressure changes measured by the PSP highlights cycle-to-cycle variations in pressure at the tip of the retreating blade.

\section{Experimental apparatus}

A small-scale helicopter was mounted in a low-speed wind tunnel to simulate forward flight conditions. The details of the helicopter model, paint characteristics, and PSP instrumentation are described in this section.

\subsection{Equipment}

The experiments were conducted in the Battelle Subsonic Wind Tunnel Facility at the Ohio State University Aeronautical and Astronautical Research Laboratories. The Eiffel wind tunnel is capable of producing test section speeds up to $45 \mathrm{~m} / \mathrm{s}$ with a belt-driven $93.2 \mathrm{~kW}(125 \mathrm{hp})$ AC motor. The test section is $0.9 \mathrm{~m}$ high, $1.5 \mathrm{~m}$ wide, and $2.9 \mathrm{~m}$ long. Four screens and a honeycomb in the settling chamber condition the flow, while an area contraction of 6.7 further reduces turbulent velocity fluctuations.

A schematic of the setup and instrumentation is shown in Fig. 2. The rotation plane of the $0.256 \mathrm{~m}$ diameter rotor was approximately $35 \mathrm{~cm}$ from the floor of the test section (1.4 rotor diameters); the center of the hub was nearly 6 rotor diameters away from the closest sidewall and 2.2 rotor diameters from the test section ceiling. Optical equipment was installed on a platform above the ceiling window. Illumination was provided by a pulsed $\mathrm{Nd}$ : YAG laser (New Wave Solo 200XT, $532 \mathrm{~nm}$ wavelength, $200 \mathrm{~mJ} /$ pulse) with a maximum repetition rate of $15 \mathrm{~Hz}$. The laser beam was expanded by a spherical divergent lens ( $-50 \mathrm{~mm}$ focal length), and the resulting light volume was smoothed by a glass diffuser.

Light emitted by the PSP (wavelength peak at $650 \mathrm{~nm}$ ) was recorded by a scientific-grade CCD camera (Cooke Corp. pco.1600) with pixel depth of 14 bits. Two $590 \pm 6 \mathrm{~nm}$ optical long-pass filters (Schott OG590 colored glass) were attached in front of the camera to reject excitation light. The interline transfer architecture of the camera allowed double-exposure images to be acquired in quick succession, an option commonly used with particle image velocimetry systems. With an 85-mm lens on the camera and $2 \times 2$ pixel binning, the spatial resolution was approximately 6 pixels per mm (575 pixels across the painted blade span and 140 pixels across its maximum chord) and the camera frame rate was $6.6 \mathrm{~Hz}$. The timing

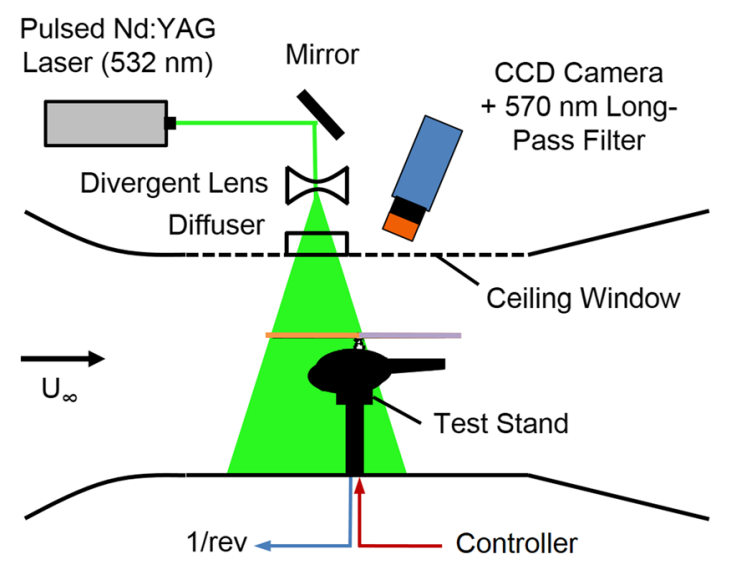

Fig. 2 Schematic of helicopter setup in wind tunnel 
system was triggered by a once-per-revolution (1/rev) signal provided by the output of a motor speed controller. Note that the camera simply ignores trigger signals before it is ready to record the next image. A pulse/delay generator (Quantum Composer Model 9614) received an exposure trigger from the camera to flash the laser.

\subsection{Helicopter model}

A hobby helicopter kit (Dynam E-Razor 250) with articulated blade control mechanism was procured for wind tunnel testing. The model was mounted on a stand in the test section by fastening the fuselage skids to a bracket as shown in Fig. 3.

The main rotor consisted of two Electrifly V-Pitch blades with a radius $R=12.8 \mathrm{~cm}$. These untwisted, rectangular blades were substituted for the longer-span blades originally packaged with the helicopter kit due to a structural resonance frequency in the rotational degree of freedom which was encountered after installation on the test stand. The longer blades had limited the maximum tip speed in hover to $<40 \mathrm{~m} / \mathrm{s}$, which would have challenged the pressure response limit of PSP. The shorter blades allowed much higher tip speeds to be reached without any signs of structural resonance. These considerations are particular for the current low-speed, small-scale setup. While a detailed trim procedure was not carried out for the helicopter model, the blade hinge screws were carefully tightened to an appropriate torque for the model to operate with minimal vibration of the motor shaft while balancing any mass difference between the painted blades.

A detailed view of the articulated hub mechanism is also shown in Fig. 3. Stabilizer wings ("fly bars") attached to the main rotor shaft help dampen disturbances to the system while spinning and can be seen below the blade hinges in the

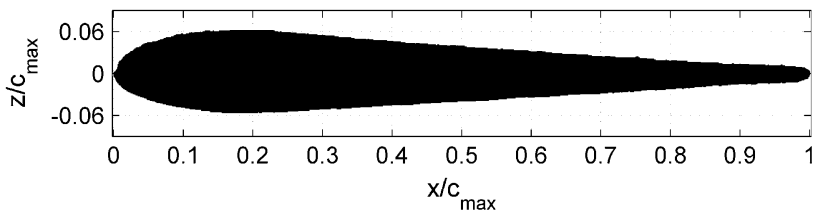

Fig. 4 Airfoil profile for hobby helicopter blade

photograph. Note that the hub does not include a flapping hinge, only a drag hinge in the lead/lag direction. The blade pitch angle $\alpha$ and rotation rate $f$ were commanded by inputs to a LabVIEW Virtual Instrument which communicated with the on-board controller and servo motor; the servo was connected to the swashplate via three pitch links. For forward flight conditions, the collective pitch was set to $10^{\circ}$ (the maximum height to which the swashplate could be raised) with longitudinal cyclic pitch of $2.5^{\circ}$. A large collective setting was desired in order to ensure a strong pressure signal which the PSP could resolve; this was a delicate requirement given the proximity of the collective angle to typical values of static stall angle. In hover, the collective pitch was set to $10^{\circ}$ with no cyclic. No thrust measurements were acquired for the purposes of this work.

The symmetric airfoil profile for the hobby helicopter blades is shown in Fig. 4, indicating a maximum thicknessto-chord ratio of approximately $12 \%$. Each blade was fabricated from fiber-reinforced nylon.

The rotor was spun at its maximum rate of $f=82 \pm 0.5 \mathrm{~Hz}$, which was limited by the maximum current draw on the unregulated power supply used as a substitute for on-board battery power. To simulate forward flight conditions, the test section speed $V_{\infty}$ was set to a fraction of the tip speed $U_{\text {tip }}=2 \pi f R$ in the plane of rotation; the ratio of these two speeds is defined here as the advance ratio $\mu$ :
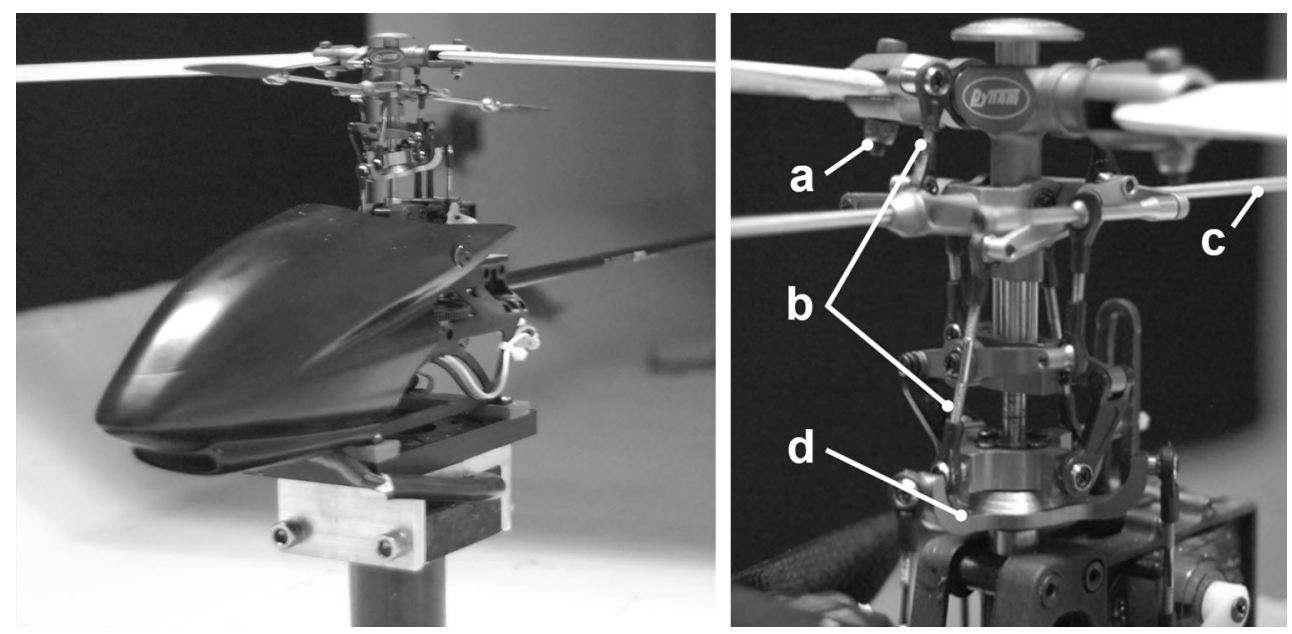

Fig. 3 Left photograph of model helicopter installed in wind tunnel. Right $\mathbf{a}$ blade hinge, b pitch links, $\mathbf{c}$ stabilizer bar, and $\mathbf{d}$ swashplate on rotor hub 
Table 1 Rotor test properties

\begin{tabular}{ll}
\hline Blade chord & $2.5 \mathrm{~cm}$ \\
Rotor disk radius & $12.8 \mathrm{~cm}$ \\
Drag hinge location & $0.19 R$ \\
Number of blades & 2 \\
Blade mass (unpainted) & $3.96 \mathrm{~g}$ \\
Blade mass (with PSP) & $4.31 \mathrm{~g}$ \\
Rotor solidity & 0.097 \\
Average power supplied & $120 \mathrm{~W}$ \\
Rotation rate & $82 \pm 0.5 \mathrm{~Hz}$ \\
Hover tip speed & $66 \pm 0.4 \mathrm{~m} / \mathrm{s}$ \\
Hover tip Mach number & 0.19 \\
Cycle-averaged $R e_{\mathrm{c}}$ at tip & $1.1 \times 10^{5}$ \\
Commanded pitching motion & $\mu=0: \alpha=10^{\circ}$ \\
& $\mu \neq 0: \alpha=10^{\circ}+2.5^{\circ} \cos (\psi)$ \\
\hline
\end{tabular}

$\mu=\frac{V_{\infty}}{\Omega R}$

where $\Omega=2 \pi f$ is the radian frequency of the rotor in radians per second. A complete listing of the rotor test properties is provided in Table 1. Note that the cosine pitching motion is generally employed for aerobatic models used by hobby enthusiasts; thus, the helicopter controller was pre-programmed for this motion.

The chord-based Reynolds number is defined as $R e_{\mathrm{c}}=U c / v_{\infty}$, where $U$ is the local relative velocity in the plane of rotation, $c$ is the airfoil chord, and $v_{\infty}$ is the kinematic viscosity of air at ambient conditions; the cycleaveraged value of $R e_{\mathrm{c}}$ at the blade tip was 110,000 and the tip Mach number in hover was 0.19. Throughout this work, the azimuthal angle $(\psi)$ convention follows the depiction in Fig. 5, with the downstream azimuth position being $\psi=0^{\circ}$. The $\psi=90^{\circ}$ azimuth is on the advancing blade side of the rotor disk, while the $\psi=270^{\circ}$ position is on the retreating blade side.

\subsection{Paint characteristics}

Two optical paints were employed on the rotor: fastresponding PSP and conventional TSP. The upper surface of one blade was painted with PSP while TSP was applied to the upper surface of a second blade. TSP is similar to conventional polymer-based PSPs, except that the temperature-sensing luminescent molecules are encapsulated in an oxygen-impermeable binder; PSP binders, on the other hand, must be permeable to oxygen.

\subsubsection{Pressure-sensitive paint}

The paint employed by Juliano et al. (2011) on a hovering rotor consisted of bathophen ruthenium chloride, $\mathrm{Ru}(\mathrm{dpp})$,

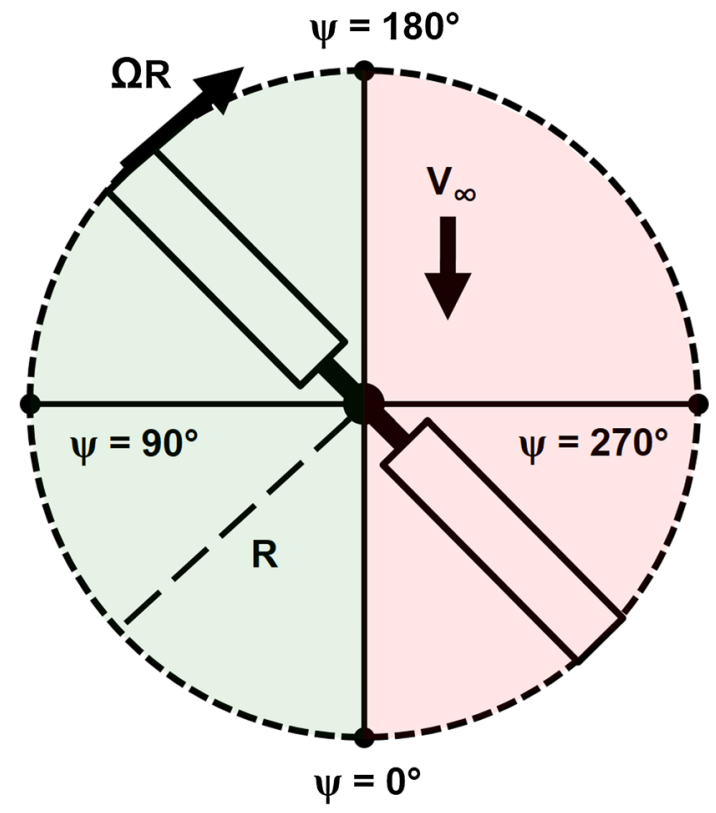

Fig. 5 Blade azimuthal angle convention

deposited in RTV-118 silicone doped with silica gel particles and was chosen for its high pressure sensitivity $(0.81 \%$ change in intensity per $\mathrm{kPa})$. However, the RTV118 binder has a very low rate of oxygen diffusion which directly leads to a poor frequency response on the order of a few $\mathrm{Hz}$ for thin paint layers and even less for thicker layers (Winslow et al. 1996). This property is inconsequential for the quasi-steady, axisymmetric flow of a hovering rotor. A PSP with much higher frequency response is necessary for unsteady measurements on a rotor in forward flight, which includes flow phenomena such as stalling of the retreating blade and flow reattachment on the advancing blade (Leishman 2006). Paint binders consisting of a porous matrix, such as PC or anodized aluminum, have been shown to exhibit fast response times by increasing the diffusion coefficient of the layer (Sakaue et al. 2002; Gregory et al. 2008, 2014). The PC base can be airbrushed onto the test surface, whereas anodized aluminum requires electrolytic treatment of the model substrate. For this reason, a PC binder was chosen for application.

The luminescent lifetime is an important factor is achieving high pressure sensitivity. For $\mathrm{Ru}(\mathrm{dpp})$ in PC, the lifetime is approximately $7.85 \mu$ s (Juliano et al. 2011). PtTFPP in PC, with a longer lifetime of approximately $11 \mu \mathrm{s}$, is more than three times as sensitive as $\mathrm{Ru}(\mathrm{dpp})$ in $\mathrm{PC}$, resulting in a pressure sensitivity of approximately $0.7 \%$ per $\mathrm{kPa}$ at standard atmospheric conditions (Sakaue et al. 2002; Gregory and Sullivan 2006; Gregory et al. 2006, 2007). The $-3 \mathrm{~dB}$ roll-off frequency for PtTFPP in $\mathrm{PC}$ has been determined to be approximately $6.5 \mathrm{kHz}$ (Sugimoto et al. 2012; Peng et al. 2013). Thus, PtTFPP in 
PC was chosen as the most desirable paint for these unsteady tests due to its high sensitivity, ease of application to the blade, and its enhanced frequency response. It must be noted that the long lifetime of this paint can cause blurring in the images if the model is moving too rapidly relative to the camera, which introduces error in the measured pressure distribution and corrupts data near the leading and trailing edges. The post-processing deblurring filter described in the data acquisition and processing section is aimed at correcting this error.

\subsubsection{Temperature-sensitive paint}

The PSP consisting of PtTFPP in PC binder has been shown to exhibit a relatively high degree of temperature sensitivity-over $3 \%$ intensity change per kelvin (Juliano et al. 2012). The temperature gradient can become a major source of bias error in the presence of local heat transfer. The temperature effects can entirely overwhelm the PSP signal, especially when the pressure change on the blade is small. To correct for the bias error introduced by temperature gradients, TSP was applied to a second blade on the main rotor. A white screen layer of spray paint was applied to the blade before TSP application to hide surface marks and increase the amount of light reflected toward the camera. Ru(bpy) in DuPont ChromaClear HC-7776S served as the TSP, which had sufficient temperature sensitivity (approximately $2 \%$ decrease in intensity per degree $\mathrm{K}$ increase) to detect the gradient of nearly $2 \mathrm{~K}$ from hub to tip expected in this work; this is discussed further in the next section. The 532-nm laser illumination source is a less-efficient excitation wavelength for the TSP, but it was necessary to make use of these sub-optimal TSP conditions in order to quickly measure the temperature field during a test. The peak emission of this TSP is at $588 \mathrm{~nm}$, while the lifetime is approximately $5 \mu \mathrm{s}$-less than half of the PSP lifetime in this work (Liu and Sullivan 2005). The TSP images showed negligible signs of the blurring problem unlike the longer-lifetime PSP and thus did not require further treatment.

\section{Data acquisition and processing}

The data acquisition rate for the single-shot mode described in this work is limited by the low repetition rates of the camera and laser (maximum near $30 \mathrm{~Hz}$ ). With a rotor frequency above $80 \mathrm{~Hz}$, this means that the pressure data were not acquired in consecutive cycles; in this work, the effective data acquisition rate corresponded to one image pair every thirteenth rotor revolution.

In theory, all of the wind-on pressure information can be obtained from a single ratio of the two exposure gates within a single lifetime, without requiring a true wind-off reference. The lifetime of the paint at a uniform pressure and temperature is ideally constant across the surface. However, in practice, inhomogeneity in the paint application and composition cause point-to-point variations in the calibration (Goss et al. 2000, 2005). A separate wind-off reference can be used to eliminate these spatial variations. The wind-off reference is also a ratio of two images from one illumination pulse, and the wind-on ratio is mapped to this reference through the image registration process described in this section. Thus, the wind-on and wind-off image pairs are used to form a ratio-of-ratios for each pixel:

$\frac{I_{\mathrm{ref}}}{I}=\frac{\left(I_{2} / I_{1}\right)_{\mathrm{ref}}}{I_{2} / I_{1}}$

where $I$ is the measured light intensity, $\left(I_{2} / I_{1}\right)_{\text {ref }}$ is the ratio of exposure gates acquired at wind-off conditions and $I_{2} / I_{1}$ is the ratio of exposure gates with an unknown pressure change present. In this way, pixel-to-pixel variations in the paint calibration are removed in the ratio-of-ratios. The emitted light intensity was calibrated to pressure using the Freundlich model for porous paints (Liu and Sullivan 2005),

$\frac{I_{\text {ref }}}{I}=A(T)+B(T)\left(\frac{p}{p_{\text {ref }}}\right)^{\gamma}$

where $p$ is the local pressure at wind-on conditions and $p_{\text {ref }}$ is the reference pressure at wind-off conditions, equal to ambient pressure $p_{\infty}$. $A, B$, and $\gamma$ are empirical coefficients which are functions of temperature $(T)$, determined from an a priori static PSP calibration over a range of pressure from 76 to $131 \mathrm{kPa}$ and temperature range between 283 and $303 \mathrm{~K}$. The surface pressure at each point on the blade was computed from the measured intensity and temperature fields using a third-order surface fit, $p=p\left(I_{\text {ref }} / I, T\right)$, to minimize error in a least-squares sense. The single-shot TSP images were processed in the same way as the PSP images, with the exception that a linear calibration was used. The relationship between temperature and TSP intensity is given as

$\frac{I_{\mathrm{ref}}}{I}=C+D \frac{T}{T_{\mathrm{ref}}}$

where $T$ is the local temperature at wind-on conditions and $T_{\text {ref }}$ is the temperature at wind-off conditions, equal to ambient temperature $T_{\infty}$. The empirical coefficients $C(5.998)$ and $D(-4.998)$ were determined from an a priori static TSP calibration over the range of temperature from 292 to $304 \mathrm{~K}$.

The wind-off reference images were acquired prior to spinning the rotor to eliminate transient temperature effects that may be present as the rotor cooled down. Ambient light effects such as natural light entering the test section 
were removed by subtracting "tare" images which were captured with the laser illumination turned off. This was done for both wind-on and wind-off conditions.

A series of post-processing steps were carried out on the acquired images, as described below. All image processing steps were accomplished with MATLAB R2013a (The MathWorks, Inc.) and its Image Processing Toolbox.

\subsection{Deblurring filter}

A deblurring tool developed by Juliano et al. (2012) was implemented in MATLAB. The Image Processing Toolbox includes built-in functions that deconvolve an image with a point-spread function describing the motion blur pattern in order to produce a blur-free image. Note that the deblurring is a function of the local paint lifetime, which in turn is a function of pressure and temperature. For very large pressure and temperature gradients on the blade (such as a shock wave), an iterative deblurring scheme may be required in some instances. In the present experiments, wherein the maximum pressure change was approximately $10 \%$ of atmospheric pressure and the maximum temperature change was near $2 \mathrm{~K}$, this additional step was found to be unnecessary.

Figure 6 shows the wind-on Gate 2 image of the rotor blade with dark levels subtracted; a rectangle is drawn around the black dots near the tip to highlight the most noticeable blurred features. The leading edge is at the top of the figure and the hub is toward the right; the rotation direction is clockwise. Figure 7 shows the deblurred version of Fig. 6. The crisp edges of the blade are recovered in the deblurred image, resulting in improved data accuracy near the blade edges. In addition, the well-defined fiducial markers aid the correlation process during image registration.

\subsection{Image registration}

It is critical for the wind-on and wind-off images to be properly aligned to within half of a pixel (Bell and McLachlan 1996). To accomplish this, the wind-on Gate 2 image was re-registered to the wind-off pair after deblurring was performed. The wind-on images were registered to the base wind-off images by selecting twelve control points on the blade (the fiducial markers shown in Fig. 7) which were applied to the blade using a felt-tip pen. This allowed for the construction of a spatial transformation using MATLAB's "projective" method, which accounts for translation, rotation, and shearing of the wind-on blade image relative to the wind-off image.

\subsection{Temperature correction}

Without correcting for the temperature gradient on the blade, the pressure map takes on a very different character.

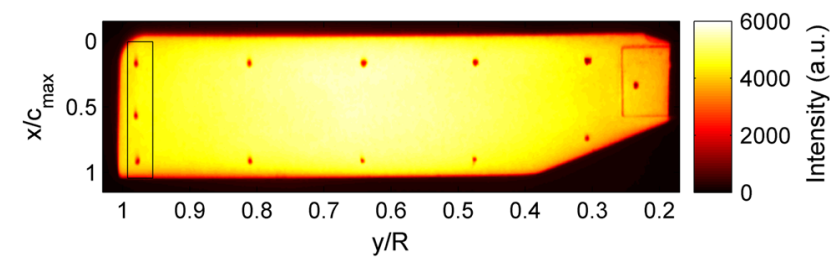

Fig. 6 Raw wind-on PSP image showing blurred fiducial markers and edges

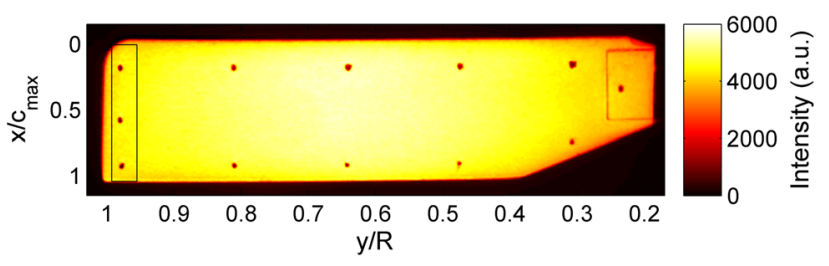

Fig. 7 Deblurred wind-on PSP image

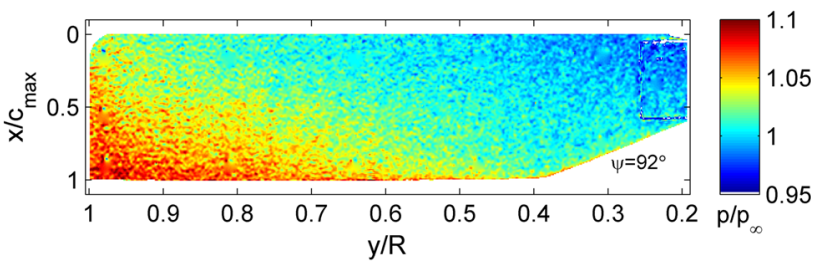

Fig. 8 Unfiltered, temperature-uncorrected pressure map for hovering rotor $\left(7.5^{\circ}\right.$ collective $)$

Figure 8 presents an example of a typical temperatureuncorrected blade pressure map from the experiments with the deblurring filter having already been applied. No spatial filtering was applied to the PSP data in

Figure 8 to demonstrate the signal level achieved with the single-shot technique; only the fiducial markers for image registration were filled in using MATLAB's "roifill" tool. Note that the pressure appears to increase from hub to tip $(y / R=1)$ and exceeds ambient at the outboard stations. This misleading result is a direct consequence of the radial temperature gradient producing false pressure information.

The expected temperature field may be estimated using the adiabatic wall recovery temperature, which is a good approximation for this case due to the low thermal conductivity of PC PSP (Egami et al. 2013). For this discussion, a hover tip speed of $66 \mathrm{~m} / \mathrm{s}$ from the experiment will be used with an ambient air temperature and pressure of $296 \mathrm{~K}$ and $101.325 \mathrm{kPa}$, respectively. The adiabatic wall recovery temperature can be calculated as:

$T_{w}=T_{\infty}\left[1+r(\operatorname{Pr})\left(\frac{k-1}{2}\right) M^{2}\right]$ 
where the recovery factor $r$ (function of Prandtl number $P r$ ) is approximately 0.85 for a laminar boundary layer with $\operatorname{Pr}=0.72$, the Mach number $M$ at the blade tip is 0.19 , and the ratio of specific heats $k$ is 1.4 (Anderson 2007). With these values, the wall recovery temperature at the blade tip is $297.8 \mathrm{~K}$, which represents a maximum change in temperature of $1.8 \mathrm{~K}$ relative to ambient. Note that the temperature increases with the square of Mach number, so that the blade temperature should vary quadratically from a minimum value at the hub to a maximum value at the tip. Moreover, the temperature field on the PSP blade can be considered quasi-steady; the timescale of the once-perrevolution unsteadiness is two orders of magnitude longer than the convective timescale of the mean flow. In addition, the PC PSP layer has been shown to have a large heat capacity, such that temperature fluctuations inside the paint layer are orders of magnitude smaller than those occurring above the paint layer (McGraw et al. 2003; Sakaue 2003). Thus, it was acceptable to measure average temperature changes on the blade with TSP and to use the hover temperature field to reduce the PSP data in forward flight.

Juliano et al. (2012) measured the blade temperature on a steady rotor with an infrared (IR) camera. Though effective, its drawbacks include having an optical window which transmits IR wavelengths (such as a sapphire window) and the blade images are prone to blurring from typically long exposures. For these reasons, it was decided in this work to adopt TSP for the temperature correction. A sample TSP temperature field acquired while the rotor was operating in the hover state is shown in Fig. 9, in which a 13-pixel radius averaging disk was used to spatially filter the data. The TSP detected a spanwise gradient with negligible chordwise variation.

By making use of the radial nature of the blade temperature field (cf. Fig. 9), a spanwise TSP profile was extracted along $x / c_{\max }=0.25$ and a second-order polynomial was fitted to the data. Figure 10 shows a representative profile at quarter-chord from the TSP data acquired in hover $\left(7.5^{\circ}\right.$ collective). At an increased collective of $10^{\circ}$, the temperature map was nearly identical. The expected quadratic dependence of blade temperature with radial position $(M \sim \Omega y)$ is readily verified from the profile. Moreover, the theoretical wall temperature at the blade tip agrees with the TSP measurement to within $0.1 \%$.

An approximated temperature map was generated which could be overlayed on the PSP data for correcting the raw intensity field. This reduced the level of noise which would be introduced by overlaying the TSP data directly onto the PSP data for a point-by-point correction, especially due to errors caused by registering the TSP blade to the PSP blade. An example temperature map produced from the curve fit is shown in Fig. 11, which reflects the radial nature of the distribution.

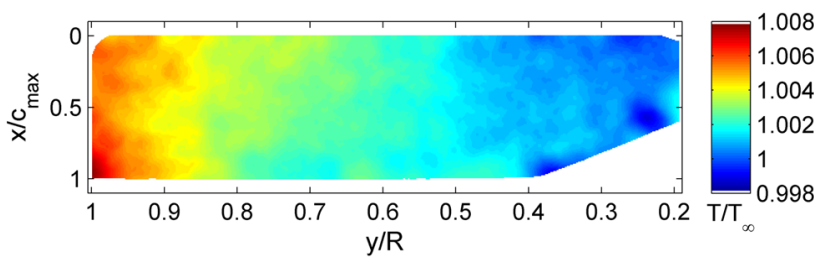

Fig. 9 Single-shot temperature map from hovering rotor $\left(7.5^{\circ}\right.$ collective)

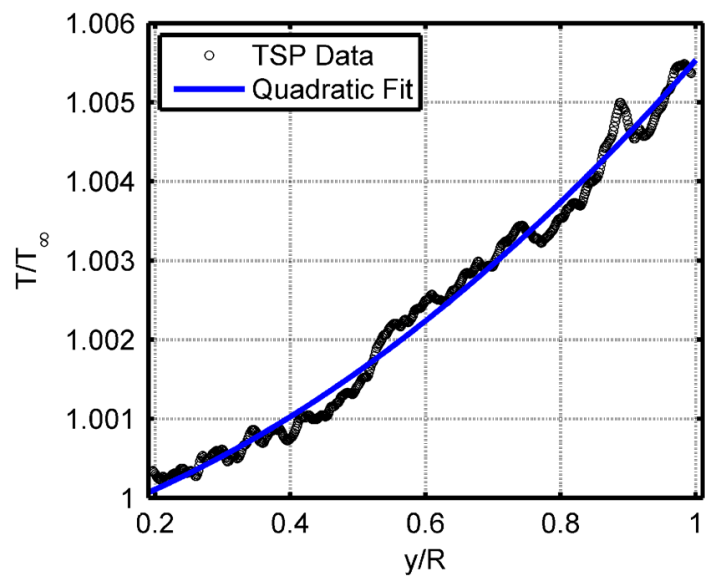

Fig. 10 Spanwise curve fit to temperature data along $x / c_{\max }=0.25$

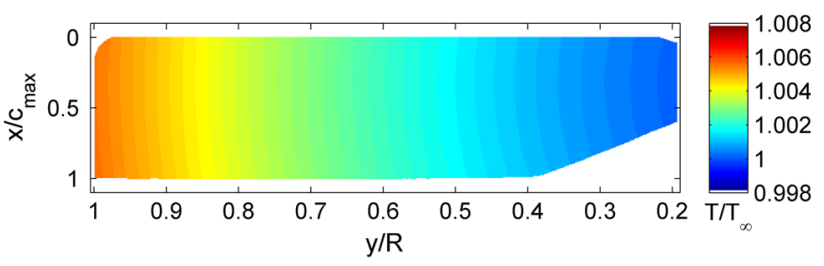

Fig. 11 Approximated temperature map produced from curve fit to TSP data

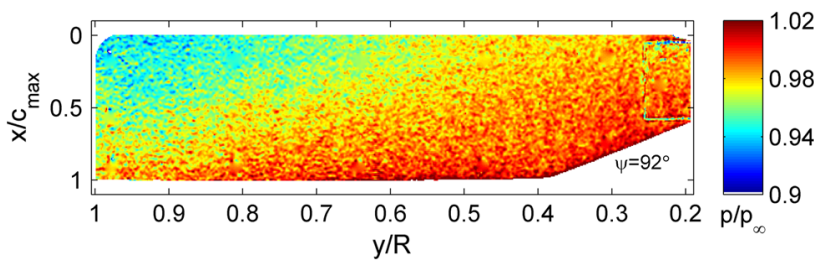

Fig. 12 Temperature-corrected, unfiltered pressure map for hovering rotor $\left(7.5^{\circ}\right.$ collective $)$

With the temperature field in hand, a static a priori calibration was applied to the PSP data to determine surface pressure based on the raw emitted light intensity and local temperature at each point. An example pressure map for the hovering rotor with temperature correction applied is shown in Fig. 12; the pressure map was left spatially unfiltered here in order to provide a visual reference for the signal-to-noise ratio in a single-shot image. The expected 
trend of leading-edge suction followed by pressure recovery toward the trailing edge is faithfully represented in the temperature-corrected PSP data.

\subsection{In situ shift}

Near the trailing edge of the temperature-corrected pressure maps for all cases, it was observed that the pressure ratio $p / p_{\infty}$ exceeded a value of unity even after the temperature correction. This was often beyond any uncertainty in the measurement of ambient pressure; in fact, the trailing edge pressure values were monitored to steadily increase with frame number, indicating a bulk shift of the mean pressure level. Following a detailed data analysis, it was concluded that the bulk increase largely occurred during the wind-on data acquisition. Increasing bulk blade temperature and/or photodegradation issues due to high-energy laser excitation are the likely causes of this bulk shift, and a suitable correction was necessary to remove the trend. In previous work by Disotell and Gregory (2011), the symptoms of photodegradation were addressed by comparing a reference field acquired before and after the wind-on data acquisition to compute a relative measure of the mean signal reduction. Their acoustic experiment did not have appreciable temperature effects as the current work does; differences in blade temperature before spinning and after spin-down complicate the matter, making it difficult to distinguish between temperature and photodegradation effects. The normal practice in PSP data reduction is to perform an in situ adjustment to the calibration based on available pressure taps. Without any pressure taps on the thin blade in this work, a trailing-edge $C_{\mathrm{p}}=0$ condition was enforced as a first-order correction method for these particular bias errors. Use of this condition is justified as follows.

Brown and Daniels (1975) considered the problem of a pitching plate in a uniform, incompressible flow and its theoretical consequences for satisfying an unsteady Kutta condition. They reasoned that for values of Strouhal number $S t=\Omega \mathrm{c} / V_{\infty}$ of $O\left(<R e_{\mathrm{c}}^{1 / 4}\right)$, the flow near the trailing edge could be considered quasi-steady. For both advance ratios in the current work, this condition is satisfied. Ohashi and Ishikawa (1972) reported a similar finding in their low-Reynolds number flow visualization of plunging airfoils, where a dividing streamline always appeared to leave the trailing edge. Furthermore, in the case of separated flow occurring near the trailing edge, the flow behind the separating streamline is approximately at ambient pressure (Crighton 1985). Zumwalt and Naik (1977) achieved good agreement between experiment and their computational model of the separated flow over an airfoil by matching the pressure at the separation point to the pressure at the lower surface trailing edge point.
With this insight into the trailing edge region, a bulk in situ shift was extracted from the trailing edge pressure on a frame-by-frame basis to remove the bias. This procedure was also adopted by Juliano et al. (2011, 2012). It is emphasized that the in situ shift is not an in situ calibration, as no sensors were available on the blade. A rectangular patch along the inboard trailing edge of the blade-where the local velocity is relatively lower than the outboard stations-was selected in each frame to extract the shift value. The rectangular patch was limited to a height of 2 pixels in the chordwise direction (1.3\% of blade chord) and covered approximately $20 \%$ of the inboard span. The in situ shift calculated the difference of the median value in this patch from unity. This difference was then treated as a bulk shift which was subtracted from the pressure ratio at each pixel on the blade.

\subsection{Spatial filtering}

A symptom of using an interline transfer CCD camera with the single-shot PSP technique is the appearance of vertical stripes in the images, as detected by Kumar (2010). The stripes are caused by the opening of transfer gates on the sensor chip which begins the readout process for the first exposure while the second frame is exposing. This momentarily creates an effectively larger pixel due to changes in the electrical and optical properties of the chip near the transfer gates (Rappl 2013, personal communication). A post-processing method to remove this "transfer pattern" was developed using a simple notch filter which operates on the raw images row-by-row. The spatial frequency of the vertical stripes depends on the particular sensor design and allows one to determine an appropriate stopband by visual inspection of the result; here, the vertical stripes appeared every 48 pixels across the image. A second-order Butterworth filter applied to both image pairs (wind-on and wind-off) was sufficient to remove the stripes without introducing ringing artifacts.

Smoothing of the final pressure map was carried out using a moving average disk which also preserved information near the edges of the blade. The edge-preserving filter carefully smoothes the data near the boundaries of the blade without the interference of background pixels entering into the local average. This was achieved by padding the neighboring pixels away from the blade with their nearest values from the blade edges, and then carrying out the disk filtering operation. It is important to note that while the images were spatially filtered, the images themselves were not ensemble-averaged to improve the signalto-noise ratio. A 10-pixel radius averaging disk was used for spatial filtering of the PSP images. 


\subsection{Uncertainty}

The notable sources of error for PSP measurements include shot noise, image registration, temperature effects, photodegradation, and pixel-to-pixel variation in the paint calibration. Using the in situ adjusted trailing edge pressure at midspan as a bulk error indicator for these effects after post-processing, the uncertainty in pressure ratio $p / p_{\infty}$ was less than $\pm 0.25 \%$ in all reported cases. The uncertainty in pressure coefficient $C_{\mathrm{p}, \mathrm{R}}$ defined in terms of hover tip speed as

$C_{\mathrm{p}, \mathrm{R}}=\frac{p-p_{\infty}}{\frac{1}{2} \rho_{\infty}(\Omega R)^{2}}$

was estimated to be \pm 0.097 . Phase jitter due to fluctuations in the rotation frequency of the motor $( \pm 0.5 \mathrm{~Hz})$ was determined to be $<2^{\circ}$ in all test cases.

\section{Results and discussion}

Single-shot pressure maps for several different azimuth positions and flow conditions are presented and discussed. Advancing and retreating blades $\left(\psi=95^{\circ}\right.$ and $\psi=270^{\circ}$, respectively) are shown at two forward speeds: a low-speed flight condition with advance ratio $\mu=0.15$ and a higher speed of $\mu=0.30$. The unsteady surface pressure fields are compared to the baseline hover case. In addition, unsteady PSP results for the $\psi=137^{\circ}$ position are presented to show the qualitative change in surface pressure compared with $\psi=95^{\circ}$; the two positions are separated in time by $1.4 \mathrm{~ms}$. The range of the color scale is maintained constant to aid visual comparisons among different rotor operating conditions, and the blade images were rotated such that the leading edge is always shown at the top of the image (blade-relative velocity in rotational plane pointing toward bottom of page).

\subsection{Hover case}

It is useful to inspect the blade pressure distribution in hover before proceeding to forward flight conditions, since the flow field in hover is azimuthally axisymmetric and can be considered a base flow for the more complicated unsteady cases. Figure 13 presents a single-shot pressure field measured by the PSP and normalized by ambient pressure. The blade collective was set to $10^{\circ}$ with no cyclic pitch.

The pressure map in hover shows the expected trend of suction near the leading edge of the blade $\left(x / c_{\max }=0\right)$ with pressure recovery toward the trailing edge. A zone of maximum suction exists near the leading edge of the tip region $(y / R>0.8)$ where the angular velocity is highest.

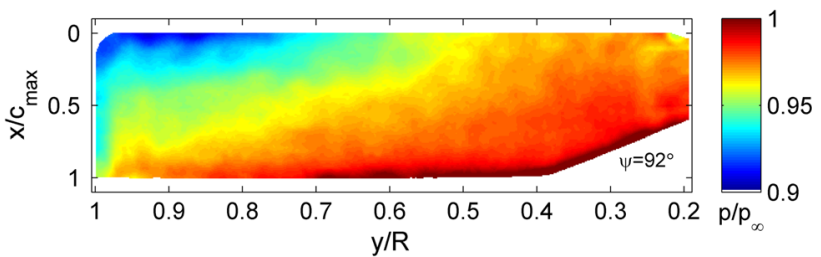

Fig. 13 Single-shot pressure map for hovering blade $\left(10^{\circ}\right.$ collective $)$

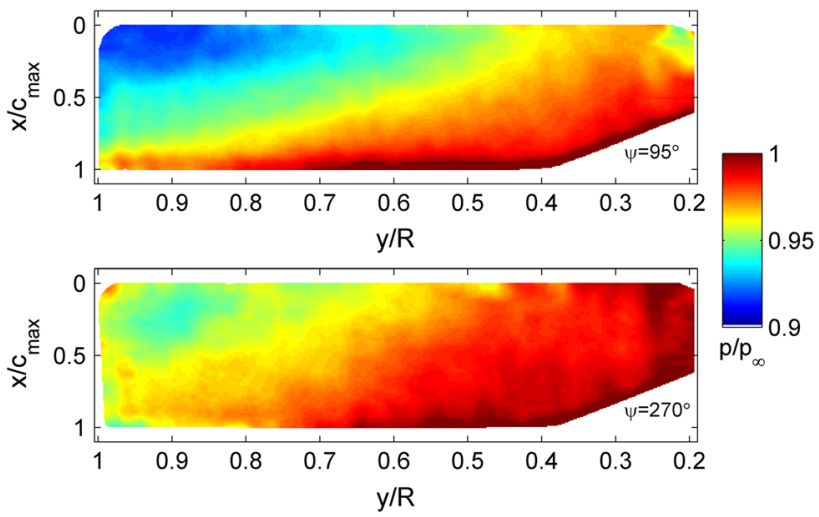

Fig. 14 Single-shot pressure map for advancing (top) and retreating (bottom) blade, $\mu=0.15$

Near the leading edge at approximately $y / R=0.22$, a suction zone appears behind the notch present in the blade geometry; this would suggest the formation of a vortex at the sharp edge. Compared with the lower collective angle of $7.5^{\circ}$ presented in Fig. 12, it is seen that the blade develops a stronger suction region at the outboard positions with increased angle of attack, as expected.

\subsection{Advancing and retreating blades in forward flight}

Single-shot pressure maps for an advancing blade $\left(\psi=95^{\circ}\right)$ and retreating blade $\left(\psi=270^{\circ}\right)$ are shown in Fig. 14 for $\mu=0.15$. The collective and longitudinal cyclic pitch on the blade were $10^{\circ}$ and $2.5^{\circ}$, respectively. The commanded pitch angle of the advancing blade was equal to that of the retreating blade for the hobby helicopter used in this work. Note that the tip edge of the retreating blade appears to be slightly canted due to the viewing angle of the blade.

The advancing blade exhibits higher suction (lower pressure) compared with the hover case due to the added component of free stream velocity relative to the blade. Although the difference in maximum pressure change from the hover case is within experimental error, the suction peak on the advancing blade is observed to be farther aft in the chordwise direction. On the retreating side, the free stream velocity relative to the blade is oriented in the opposite direction to the angular velocity. Because of the 


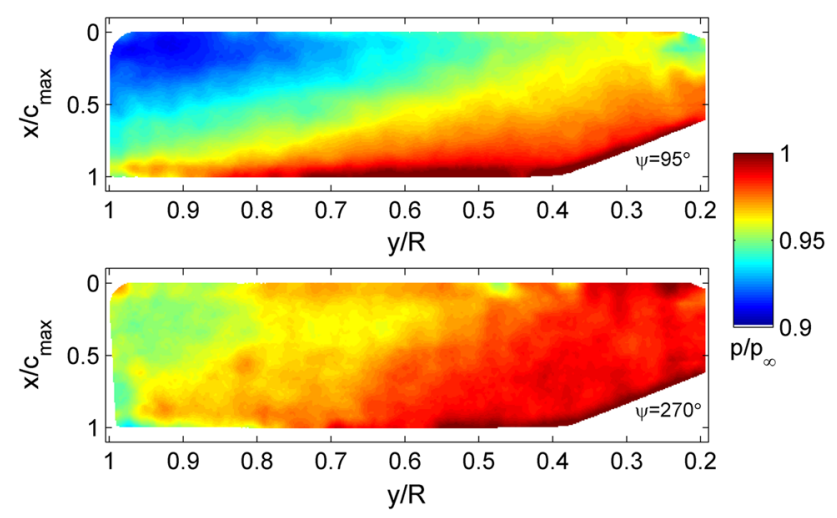

Fig. 15 Single-shot pressure maps for advancing (top) and retreating (bottom) blade, $\mu=0.30$

lower dynamic pressure seen by the retreating blade, the maximum pressure change decreased by $3.6 \%$ relative to hover. The PSP data clearly show the qualitative difference in aerodynamic environment between the two sides of the rotor disk; the hovering blade in Fig. 13 approximates the cycle-averaged blade response well. The small apparent suction features near the leading edge at $y / R=0.38$ and 0.48 on the retreating blade are artifacts attributed to paint blemishes encountered during the middle of the test campaign. These blemishes were not able to be satisfactorily filled in during post-processing due to their promixity to the leading edge; they also appear in the retreating blade data presented next.

Figure 15 presents the measured surface pressure field for the higher advance ratio of $\mu=0.30$, which corresponds to a doubling of the free stream velocity from the former case. The retreating blade exhibits a larger reduction in dynamic pressure at this increased forward speed, leading to a $4.1 \%$ decrease in maximum pressure change relative to hover.

The above results are significant in demonstrating that the PSP can resolve unsteady pressure changes on the blade, considering that the rotation rate of the rotor was nearly $82 \mathrm{~Hz}(12.2 \mathrm{~ms}$ period). This is also the fundamental frequency at which the aerodynamic loads change. As demonstrated by the pressure maps, the high spatial resolution is an inherent advantage of PSP and this allows for the chordwise pressure to be measured at every point on the surface. Figure 16 summarizes the comparisons made above by displaying instantaneous (single-shot) chordwise pressure profiles at a radial station of $y / R=0.70$ on the advancing and retreating blades for each advance ratio; the pressure coefficient $C_{\mathrm{p}, \mathrm{R}}$ for all cases is based on the same tip speed in hover. The pressure profiles indicate the aft movement of the suction peak on the advancing blade with increasing advance ratio as well as plateau regions indicative of local separation on the retreating blade. As

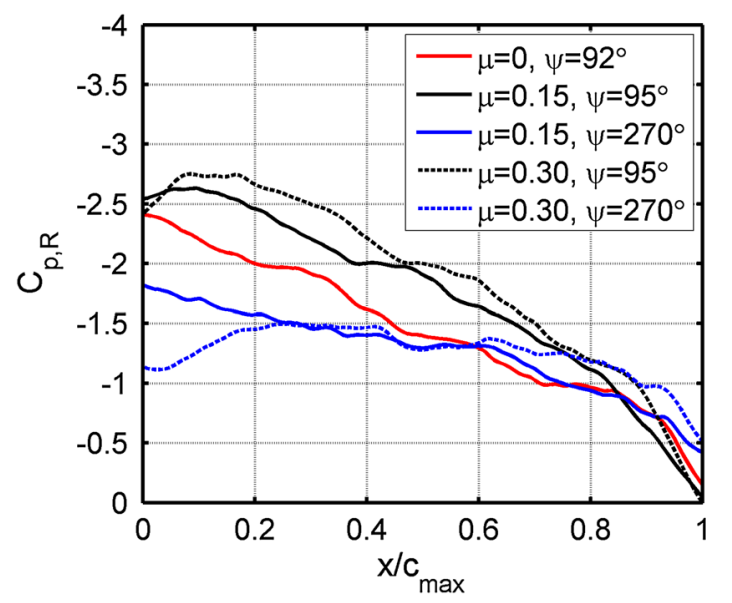

Fig. 16 Instantaneous chordwise pressure profiles at $y / R=0.70$

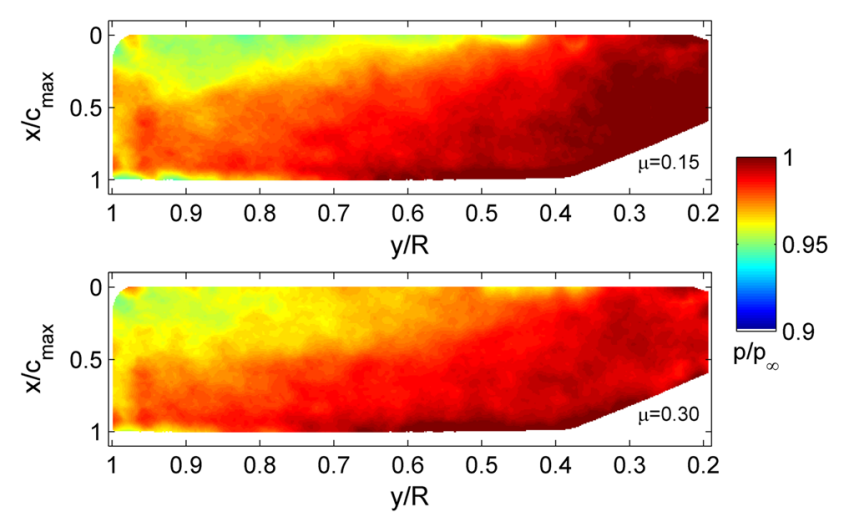

Fig. 17 Comparison of blade pressure maps for $\psi=137^{\circ}$ at two advance ratios

visualized in the single-shot PSP images, the tip region on the retreating blade does not appear to recover to the ambient pressure even after application of the in situ shift, which may be indicative of unsteady vortex dynamics. This aspect is explored at the end of the section.

\subsection{Blade with cross-flow}

To further demonstrate the fast response of PSP in the unsteady helicopter environment, the surface pressure on a blade separated in time by $1.4 \mathrm{~ms}$ from the $\psi=95^{\circ}$ position was measured. Figure 17 displays the PSP results at an azimuth position of $\psi=137^{\circ}$ for advance ratios of $\mu=0.15$ and 0.30 . At this position, the free stream flow is at a $47^{\circ}$ angle with respect to the angular velocity vector.

The blade is pitching down at this instant in the cycle to a nominal angle of $8.75^{\circ}$. It is worthwhile to note that the maximum pressure change on the blade is less than that observed on the hovering blade in Fig. 13, where the nominal blade pitch angle was $10^{\circ}$. The higher advance ratio condition appears to show off-loading at the outboard 
Fig. 18 Maps of root-meansquare pressure on advancing and retreating sides at different forward speeds

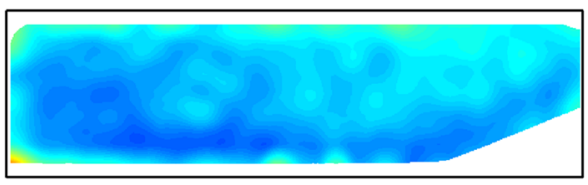

(a) $\psi=92^{\circ}, \mu=0$

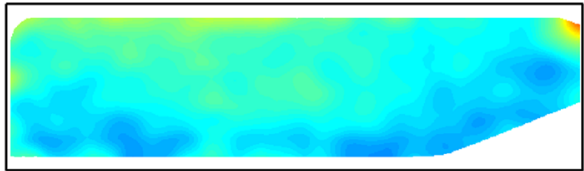

(b) $\psi=95^{\circ}, \mu=0.15$

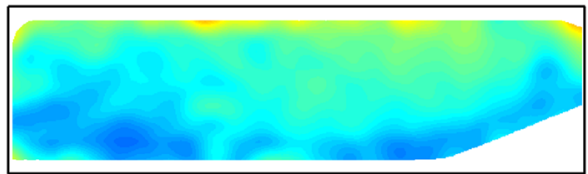

(c) $\psi=95^{\circ}, \mu=0.30$
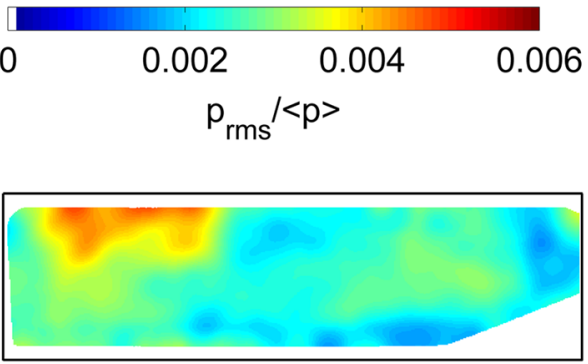

(d) $\psi=270^{\circ}, \mu=0.15$

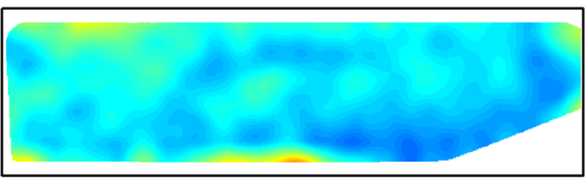

(e) $\psi=270^{\circ}, \mu=0.30$ stations compared with the low-speed flight condition, while the inboard region shows a greater pressure change arising from an increase in relative velocity. At the lower advance ratio, the inboard region appears to be largely stalled.

\subsection{Transient pressure variations}

Unsteady flow dynamics occurring on the surface of the blade are linked to coherent structures that can be monitored in a cycle-to-cycle manner using an ensemble of single-shot PSP images. The root-mean-square pressure at each surface point was normalized by the corresponding mean pressure for an ensemble of $N=25$ single-shot images. The surface pressure data were acquired every thirteenth blade revolution, ensuring sufficiently independent measurements. Figure 18 displays a comparison of the root-mean-square pressure maps between the advancing and retreating blades at several advance ratios, including hover $(\mu=0)$.

The pressure fluctuations are very low for the hover case (Fig. 18a), which is nominally steady. For the advancing blade in forward flight, the highest advance ratio (Fig. 18c) indicates higher fluctuation levels at the inboard region than the lower speed (Fig. 18b). Out of all the cases shown, the retreating blade shows the most prominent region of unsteadiness near the tip at an advance ratio of 0.15 (Fig. 18d) which is not observed at the higher speed (Fig. 18e). This is not unexpected; for example, Ghee and Elliott (1995) carried out flow visualization on the wake of a scale rotor using a laser light sheet and smoke, and they reported differences in the tip vortex structure between the advancing and retreating sides of the rotor disk which may help explain the tip unsteadiness. In their work, the tip vortices were characterized as tightly rolled-up, aligned filaments on the advancing side while those on the retreating blade side were visualized to be more diffuse. Moreover, they observed the trajectory of the shed vortices to be much shallower on the advancing side compared with the retreating side at an operating condition of $\mu=0.15$. The vortex trajectories at a higher advance ratio of $\mu=0.23$ in Ghee and Elliott (1995), however, were similar on both sides of the disk and even shallower than at the lower advance ratio. For these reasons, it is plausible that the tip unsteadiness in Fig. 18d is the signature of a vortex interaction, which greatly depends on the vortex trajectory.

To further explore the increased tip unsteadiness on the retreating blade at $\mu=0.15$, the single-shot pressure maps were converted to an AC-coupled form which displays differential pressure changes relative to the mean, $\langle p\rangle$, at each pixel (see Fang et al. 2010 for a discussion of this method). Figure 19 shows this frame-to-frame complementary view of the root-mean-square data for a subset of eight consecutive images.

Oblique pressure features are immediately visible at the outboard regions, and their structure is reminiscent of upwash/downwash from a vortex filament interacting with the surface. These features are not entirely unexpected on the retreating blade, given the strong radial layer induced by centrifugal effects near the surface. Raghav and Komerath (2013) have recently shown through particle image velocimetry (PIV) measurements that discrete structures exist in the radial flow near the surface in the stalled region. Their genesis is likely in the radial acceleration near the surface. Moreover, Raghav and Komerath (2013) found that the radial jet layer develops discrete structures at Reynolds numbers well below transition. Further investigation with coupled PSP/ PIV has the potential to be fruitful in this regard. 
Fig. 19 AC-coupled view of consecutive single-shot PSP images for $\psi=270^{\circ}, \mu=0.15$
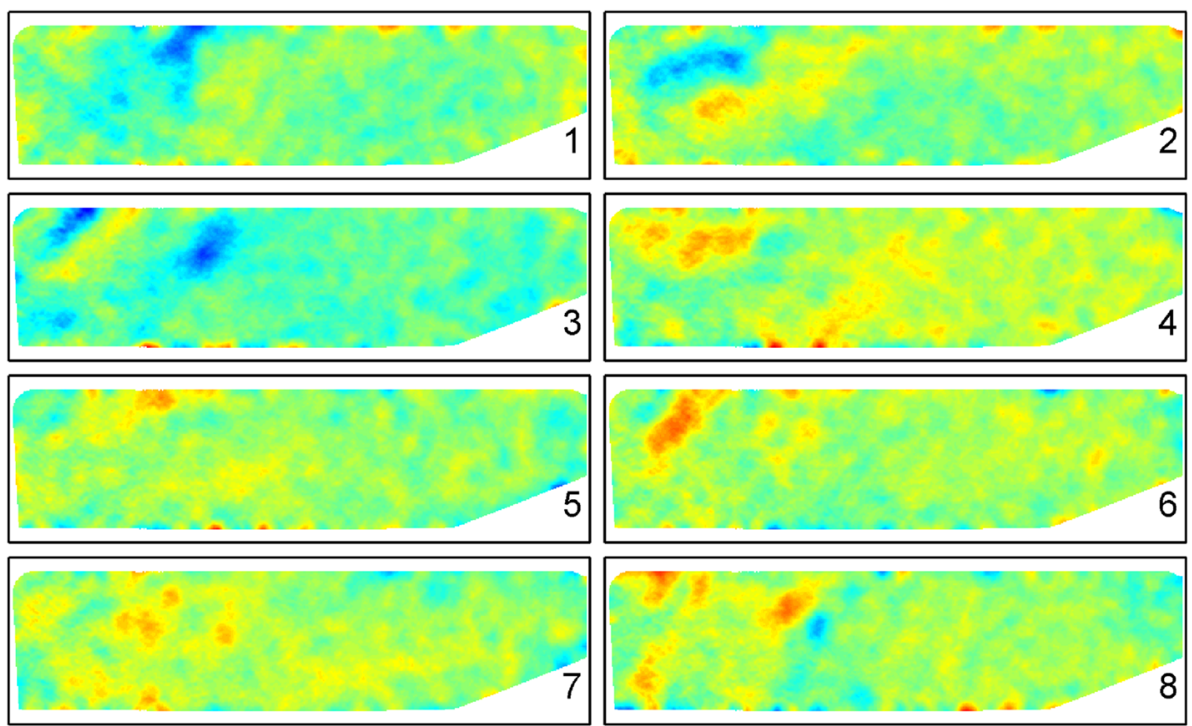

\section{Concluding remarks}

This work implemented single-shot PSP for unsteady pressure measurements on a fully articulated helicopter blade. The porous PC PSP exhibits significant temperature sensitivity, which required optical measurement of the surface temperature distribution using a second rotor blade with TSP. The measured radial temperature gradient was found to be appropriately modeled by the adiabatic recovery temperature based on local relative Mach number. In all reported cases, an increasing trend in the mean pressure was observed and attributed to bulk temperature changes and/or photodegradation. Without any sensors available on the thin blade in this work, a bulk in situ shift based on setting $C_{p}=0$ at the trailing edge was employed for first-order correction. The key contributions from this work include:

1. Examination of the measured pressure distributions on the advancing and retreating blades indicated that the paint was capable of resolving unsteady flow phenomena occurring at the rotational frequency of a smallscale, fully articulated helicopter $(82 \mathrm{~Hz})$ with fine spatial resolution. The surface pressure field was assessed at a low-speed forward flight condition with advance ratio of $\mu=0.15$ as well as a higher-speed case of $\mu=0.30$ for several blade positions, all with cyclic blade pitching.

2. The unsteady pressure data in forward flight conditions were compared to the nominally steady hover case, which represented the average blade response between

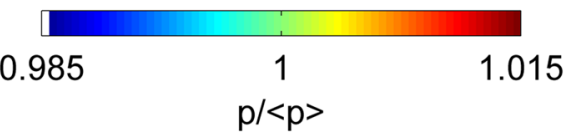

the advancing and retreating sides of the rotor disk in forward flight to a good degree.

3. The case of a blade with cross-flow on the advancing side of the disk was investigated, which revealed apparent off-loading at the highest advance ratio. Taking advantage of the capability for cycle-to-cycle monitoring of pressure variations, the retreating blade showed increased tip unsteadiness at the lower advance ratio; inspection of the differential pressure changes on a frame-by-frame basis appeared to show the signature of oblique vortex interactions possibly linked to three-dimensional stalling.

It is important to recall that the single-shot PSP images presented here are essentially independent measurements of the blade pressure due to the low repetition rates of the Nd:YAG laser and camera. With the availability of highspeed lasers and cameras, the data acquisition rate of the single-shot PSP technique can be increased to enable truly time-resolved measurements from consecutive cycles or even several azimuth positions within a single blade revolution.

Acknowledgments This article is dedicated to the late Sergey Fonov of ISSI, well-known for his contributions to the PSP technique. The authors are grateful for funding from Phase I and II SBIRs from NASA Langley Research Center, monitored by A. Neal Watkins, Luther Jenkins, and Oliver Wong. This research was also partially funded by the U.S. Government under Agreement No. W911W6-112-0010. The U.S. Government is authorized to reproduce and distribute reprints notwithstanding any copyright notation thereon. The authors also thank Matthew McCrink, Matthew Frankhouser, and Ryan McMullen for their assistance in setting up the experiment. K. J. 
Disotell acknowledges the support of a National Defense Science and Engineering Graduate Fellowship (U.S. Department of Defense) as well as a National Science Foundation Graduate Research Fellowship during this work.

Open Access This article is distributed under the terms of the Creative Commons Attribution License which permits any use, distribution, and reproduction in any medium, provided the original author(s) and the source are credited.

\section{References}

Anderson JD (2007) Fundamentals of aerodynamics, 4th edn. McGraw-Hill, New York

Bell JH, McLachlan BG (1996) Image registration for pressuresensitive paint applications. Exp Fluids 22(1):78-86

Bell JH, Schairer ET, Hand LA, Mehta RD (2001) Surface pressure measurements using luminescent coatings. Annu Rev Fluid Mech 33:155-206. doi:10.1146/annurev.fluid.33.1.155

Brown SN, Daniels PG (1975) On the viscous flow about the trailing edge of a rapidly oscillating plate. J Fluid Mech 67:743-761. doi:10.1017/S0022112075000584

Crighton DG (1985) The Kutta condition in unsteady flow. Annu Rev Fluid Mech 17:411-445. doi:10.1146/annurev.fl.17.010185. 002211

Disotell KJ, Gregory JW (2011) Measurement of transient acoustic fields using a single-shot pressure-sensitive paint system. Rev Sci Instrum 82(7):075112. doi:10.1063/1.3609866

Disotell KJ, Juliano TJ, Peng D, Gregory JW, Crafton JW, Komerath NM (2012) Unsteady pressure-sensitive paint measurements on an articulated model helicopter in forward flight. 28th aerodynamic measurement technology, ground testing, and flight testing conference, AIAA 2012-2757. American Institute of Aeronautics and Astronautics. doi:10.2514/6.2012-2757

Egami Y, Fujii K, Takagi T, Matsuda Y, Yamaguchi H, Niimi T (2013) Reduction of temperature effects in pressure-sensitive paint measurements. AIAA J 51(7):1779-1783. doi: 10.2514/1. J052226

Fang S, Disotell KJ, Long SR, Gregory JW, Semmelmayer FC, Guyton RW (2010) Application of fast-responding pressure-sensitive paint to a hemispherical dome in unsteady transonic flow. Exp Fluids 50(6):1495-1505. doi:10.1007/s00348-010-1010-1

Ghee TA, Elliott JW (1995) The wake of a small-scale rotor in forward flight using flow visualization. J Am Helicopter Soc 40(3):52-65

Goss L, Trump D, Sarka B, Lydick L, Baker W (2000) Multidimensional time-resolved pressure-sensitive paint techniques: a numerical and experimental comparison. 37th aerospace sciences meeting and exhibit, AIAA-2000-0832. American Institute of Aeronautics and Astronautics

Goss L, Jones G, Crafton J, Fonov S (2005) Temperature compensation for temporal (lifetime) pressure sensitive paint measurements. 43rd aerospace sciences meeting and exhibit, AIAA2005-1027. American Institute of Aeronautics and Astronautics

Gregory JW, Sullivan JP (2006) Effect of quenching kinetics on unsteady response of pressure-sensitive paint. AIAA J 44(3):634-645

Gregory JW, Sullivan JP, Wanis S, Komerath NM (2006) Pressuresensitive paint as a distributed optical microphone array. J Acoust Soc Am 119(1):251-261

Gregory JW, Sullivan JP, Raman G, Raghu S (2007) Characterization of the microfluidic oscillator. AIAA J 45(3):568-576

Gregory JW, Asai K, Kameda M, Liu T, Sullivan JP (2008) A review of pressure-sensitive paint for high-speed and unsteady aerodynamics. Proc Inst Mech Eng Part G J Aerosp Eng 222(2):249-290. doi:10.1243/09544100JAERO243

Gregory JW, Sakaue H, Liu T, Sullivan JP (2014) Fast pressuresensitive paint for flow and acoustic diagnostics. Annu Rev Fluid Mech 303-330. doi:10.1146/annurev-fluid-010313-141304

Juliano TJ, Kumar P, Peng D, Gregory JW, Crafton J, Fonov S (2011) Single-shot, lifetime-based pressure-sensitive paint for rotating blades. Meas Sci Technol 22(8):085403. doi:10.1088/09570233/22/8/085403

Juliano TJ, Disotell KJ, Gregory JW, Crafton JW, Fonov SD (2012) Motion-deblurred, fast-response pressure-sensitive paint on a rotor in forward flight. Meas Sci Technol 23(4):045303. doi:10. 1088/0957-0233/23/4/045303

Kumar P (2010) Development of a single-shot lifetime PSP measurement technique for rotating surfaces. M.S. Thesis, Department of Aerospace Engineering, The Ohio State University

Leishman JG (1998) Measurements of the aperiodic wake of a hovering rotor. Exp Fluids 25(4):352-361

Leishman JG (2006) Principles of helicopter aerodynamics, 2nd edn. Cambridge University Press, Cambridge

Liu T, Sullivan JP (2005) Pressure and temperature sensitive paints. Springer, Berlin

McCroskey WJ (1981) The phenomenon of dynamic stall. NASA TM-81264

McGraw CM, Shroff H, Khalil G, Callis JB (2003) The phosphorescence microphone: a device for testing oxygen sensors and films. Rev Sci Instrum 74(12):5260-5266

Ohashi H, Ishikawa N (1972) Visualization study of flow near the trailing edge of an oscillating airfoil. Bull JSME 15(85):840-847

Peng D, Jensen CD, Juliano TJ, Gregory JW, Crafton J, Palluconi S, Liu T (2013) Temperature-compensated fast pressure-sensitive paint. AIAA J 51(10):2420-2431. doi:10.2514/1.J052318

Raghav VS, Komerath NM (2013) An exploration of radial flow on a rotating blade in retreating blade stall. J Am Helicopter Soc 58(2):1-10. doi:10.4050/JAHS.58.022000

Rappl P (2013) PCO AG, Kelheim, Germany (personal communication)

Sakaue H (2003) Anodized aluminum pressure sensitive paint for unsteady aerodynamic applications. Ph.D. Dissertation, School of Aeronautics and Astronautics, Purdue University

Sakaue H, Gregory JW, Sullivan JP (2002) Porous pressure-sensitive paint for characterizing unsteady flowfields. AIAA J 40(6):1094-1098

Sakaue H, Miyamoto K, Miyazaki T (2013) A motion-capturing pressure-sensitive paint method. J Appl Phys 113(8):084901. doi:10.1063/1.4792761

Sugimoto T, Kitashima S, Numata D, Nagai H, Asai K (2012) Characterization of frequency response of pressure-sensitive paints. 50th AIAA aerospace sciences meeting, AIAA 2012-1185. American Institute of Aeronautics and Astronautics

Watkins AN, Leighty BD, Lipford WE, Wong OD, Oglesby DM, Ingram JL (2007) Development of a pressure sensitive paint system for measuring global surface pressures on rotorcraft blades. 22nd international congress on instrumentation in aerospace simulation facilities. Institute of Electrical and Electronics Engineers. doi:10.1109/ICIASF.2007.4380888

Watkins AN, Leighty BD, Lipford WE, Wong OD, Goodman KZ, Crafton JW, Forlines A, Goss LP, Gregory JW, Juliano TJ (2012) Deployment of a pressure sensitive paint system for measuring global surface pressures on rotorcraft blades in simulated forward flight. 28th AIAA aerodynamics measurement technology and ground testing conference, AIAA 2012-2756. American Institute of Aeronautics and Astronautics

Winslow NA, Carroll BF, Setzer FM (1996) Frequency response of pressure sensitive paints. 27th AIAA fluid dynamics conference, 
AIAA 1996-1967. American Institute of Aeronautics and Astronautics

Wong OD, Watkins AN, Ingram JL (2005) Pressure-sensitive paint measurements on $15 \%$ scale rotor blades in hover. 35th AIAA fluid dynamics conference and exhibit, AIAA 2005-5008. American Institute of Aeronautics and Astronautics

Wong OD, Noonan KW, Watkins AN, Jenkins LN, Yao CS (2010) Non-intrusive measurements of a four-bladed rotor in hover-a first look. Presented at the American Helicopter Society Aeromechanics Specialists Conference, San Francisco, CA, January 20-22, 2010
Wong OD, Watkins AN, Goodman KZ, Crafton JW, Forlines A, Goss L, Gregory JW, Juliano TJ (2012) Blade tip pressure measurements using pressure sensitive paint. AHS 68th annual forum and technology display, AHS 2012-000233. American Helicopter Society

Zumwalt GW, Naik SN (1977). An analytical model for highly separated flow on airfoils at low speeds. NASA CR 145249 